\title{
The First Galaxies: Assembly with Black Hole Feedback
}

\author{
Myoungwon Jeon ${ }^{* a}$, Andreas H. Pawlik ${ }^{a}$, Thomas H. Greif ${ }^{b}$, Simon C. O. Glover ${ }^{c}$, \\ Volker Bromm ${ }^{a}$, Miloš Milosavljević ${ }^{a}$, and Ralf S. Klessen ${ }^{c}$
}

${ }^{a}$ Department of Astronomy and Texas Cosmology Center, University of Texas, Austin, TX 78712, USA

${ }^{c}$ Zentrum für Astronomie der Universität Heidelberg, Institut für Theoretische Astrophysik, Albert-Ueberle-Strasse 2, 69120 Heidelberg, Germany

${ }^{b}$ Max-Planck-Institut für Astrophysik, Karl-Schwarzschild-Strasse 1, 85740 Garching bei

München, Germany

E-mail: myjeon@astro.as.utexas.edu

We study how the first galaxies were assembled under feedback from the accretion onto a central black hole $(\mathrm{BH})$ that is left behind by the first generation of metal-free stars through selfconsistent, cosmological simulations. X-ray radiation from the accretion of gas onto $\mathrm{BH}$ remnants of Population III (Pop III) stars, or from high-mass X-ray binaries (HMXBs), again involving Pop III stars, influences the mode of second generation star formation. We track the evolution of the black hole accretion rate and the associated X-ray feedback starting with the death of the Pop III progenitor star inside a minihalo and following the subsequent evolution of the black hole as the minihalo grows to become an atomically cooling galaxy. We find that X-ray photoionization heating from a stellar-mass $\mathrm{BH}$ is able to quench further star formation in the host halo at all times before the halo enters the atomic cooling phase. X-ray radiation from a HMXB, assuming a luminosity close to the Eddington value, exerts an even stronger, and more diverse, feedback on star formation. It photoheats the gas inside the host halo, but also promotes the formation of molecular hydrogen and cooling of gas in the intergalactic medium and in nearby minihalos, leading to a net increase in the number of stars formed at early times. Our simulations further show that the radiative feedback from the first BHs may strongly suppress early $\mathrm{BH}$ growth, thus constraining models for the formation of supermassive BHs.

Frank N. Bash Symposium New Horizons In Astronomy,

October 9-11, 2011

Austin Texas

\footnotetext{
*Speaker.
} 


\section{Introduction}

It has been a longstanding goal to understand the formation of the first galaxies subject to different accompanying feedback effects from the first stars, such as ionizing feedback from individual Pop III stars (e.g.,[1]; [2]), chemical feedback produced by a supernova (SN) explosion (e.g., [3]; [4]), and radiative feedback from accreting black holes (BHs) (e.g., [5]; [6]). The first stars are expected to form at a redshift $z \gtrsim 15$ inside dark matter minihalos with masses of $\sim 10^{6} M_{\odot}$ (e.g., [7]; [8]). For progenitor masses within the range of $40 M_{\odot} \lesssim M \lesssim 140 M_{\odot}$ or $M \gtrsim 260 M_{\odot}$, the Pop III star will become a massive $\mathrm{BH}$ via direct gravitational collapse. The ensuing merging of minihalos, provided they contain cold gas, and the smooth accretion of gas from the intergalactic medium (IGM) will feed the central BH. A fraction of the accreted mass-energy will then be released as radiative energy, resulting in a miniquasar (e.g., [9]; [10]), which will ionize the surrounding neutral medium and heat the pre-galactic gas.

We here briefly summarize our recent study ([11]), in which we investigated the feedback effects from such accreting stellar-mass BHs by focusing on the question: "How does a stellar black hole, a remnant of a Pop III star, influence the subsequent star formation and in turn the assembly process of the first galaxies?" Miniquasar feedback may also be expected to have important effects on BH growth. One outstanding question is how the supermassive black holes (SMBHs), observed by the Sloan Digital Sky Survey (SDSS) at $z \sim 6$ with masses of $\sim 10^{9} M_{\odot}$ (e.g., [12]), were able to grow within such a short period of time after the Big Bang. A second goal of this study is to provide an improved understanding of whether a stellar BH can grow to become a SMBH in the presence of stellar and $\mathrm{BH}$ feedback.

\section{Simulations}

To survey the relevant parameter space, we have carried out three cosmological simulations. As a reference, simulation "BHN" includes stellar radiative feedback from Pop III stars, whereas the subsequent feedback due to BH accretion is not taken into account. The simulation "BHS" includes both the feedback from Pop III stars and from a single isolated BH. Finally, in simulation "BHB" we assume that the Pop III BH remnant has a stellar binary companion, giving rise to a HMXB.

For the simulations presented here, we use a customized version of the combined hydrodynamics and N-body code GADGET2 ([13]). We start running the simulations at $z \sim 30$, corresponding to the redshift just before the first Pop III star in the computational box is formed, and terminate them at $z \sim 10$, when the assembly of the first galaxy is expected to be complete. We propagate the ionization front around it to build up a primordial H II region, using a well-tested ray-tracing algorithm (for details see [14]). We follow Kuhlen \& Madau (2005) ([5]) to model the propagation of high-energy photons emitted from the accretion onto a $\mathrm{BH}$ in which the accretion rate is estimated by the Bondi \& Hoyle (1944) model (see [15]).

\section{Results}

In Figure 1, we show the evolution of the virial mass for the most massive halo, which will host the first galaxy at $z \sim 10$, as well as of the total and cold gas masses in the three simulations 


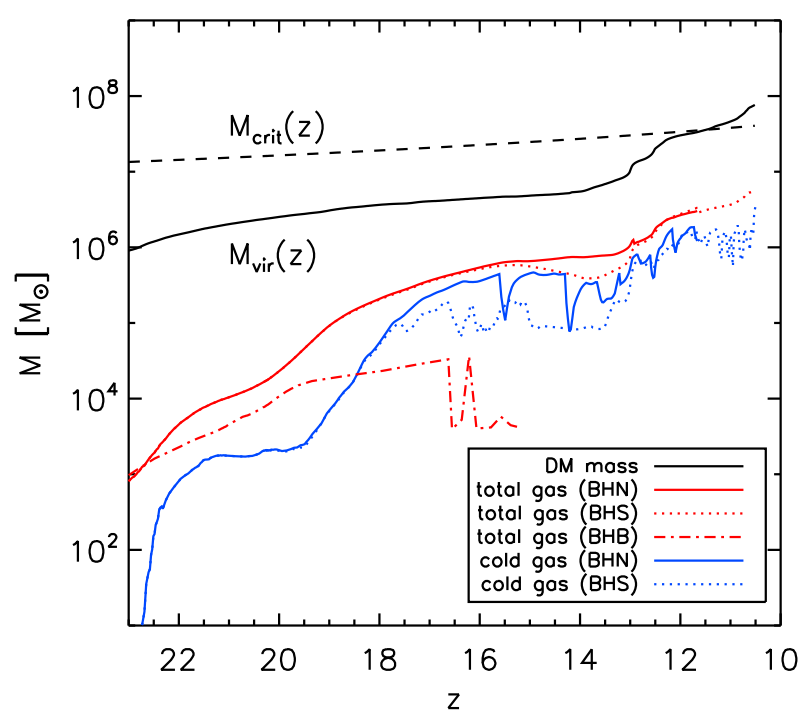

Figure 1: Assembly of the first galaxy. Shown is the redshift evolution of DM virial mass (black solid line) of the halo which will host the first galaxy at $z \sim 10$, as well as of the total gas mass (red) and the cold gas mass (blue) within the halo for simulations BHN (solid lines), BHS (dotted lines), and BHB (dot-dashed line). The black dashed line represents the critical mass required for the onset of atomic cooling in the halo at a given redshift. There is no cold gas within the halo in simulation BHB owing to the strong heating from the HMXB.

presented in this work. We find that at $z \gtrsim 18$, the halo is dominated by hot gas, exceeding the amount of cold gas by an order of magnitude. As time passes on, the cold gas mass increases, eventually accounting for $\gtrsim 80 \%$ of the total gas mass in simulations BHN and BHS. This trend can be understood by the vulnerability of the halo gas to stellar radiative feedback. The corresponding evacuation of gas from the halo is very strong at high redshifts, $z \gtrsim 18$, because the halo potential wells were not yet deep enough to retain photo-heated gas.

While the total amount of gas is not sensitive to the BH feedback, the reduction in cold gas mass by a factor of $\sim 5$ indicates that the additional heating from this feedback, on top of the stellar feedback, has a significant impact on the gas in the center of the forming galaxy. As the halo grows further via smooth accretion and mergers with minihalos, however, at $z \sim 13$, both the total gas mass and mass of cold gas are no longer sensitive to the $\mathrm{BH}$ radiative feedback.

For simulation BHB, the heating from the HMXB is so strong that all gas particles have temperatures $T>0.5 T_{\mathrm{vir}}(z)$, over the entire range of simulated redshifts $z \gtrsim 15$, and the total gas mass is reduced by nearly an order of magnitude by photo-evaporation, as is evident from Figure 1 (see the dot-dashed lines). This implies that if an HMXB existed within a minihalo at high redshifts, it would take significantly longer for the halo to reassemble the lost gas, and to eventually evolve into a primordial galaxy.

Figure 2 shows the evolution of the accretion rate onto the $\mathrm{BH}$, the density and temperature of the neighboring gas, as well as the $\mathrm{BH}$ mass for simulations BHN and BHS. The accretion rate 

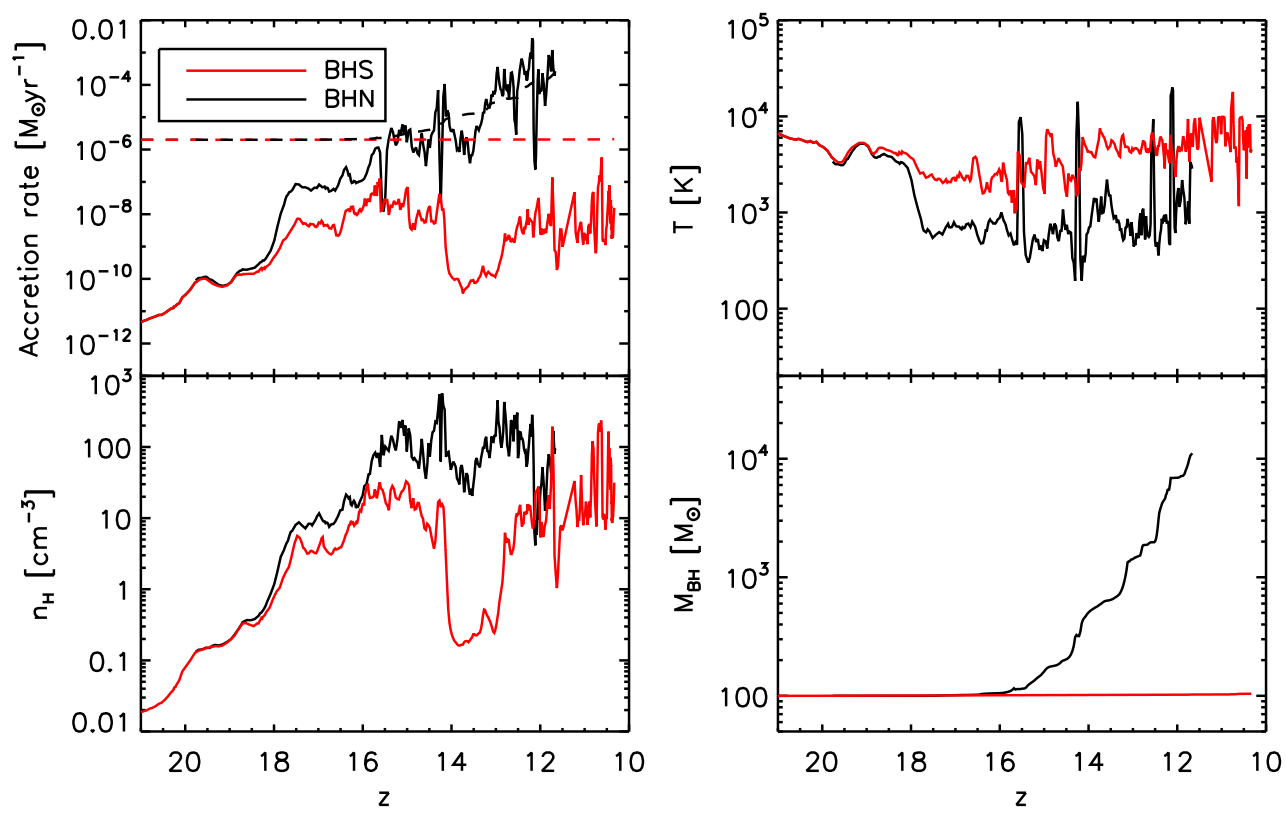

Figure 2: BH growth with and without feedback. Shown is the redshift evolution of the BH accretion rate, the density and the temperature of the gas in the immediate vicinity of the $\mathrm{BH}$, as well as the resulting $\mathrm{BH}$ masses for simulations BHN (black) and BHS (red). In the top-left panel, we also indicate the corresponding Eddington-limited accretion rates for the two cases (dashed lines).

in the BHN simulation is already comparable to the Eddington value, depicted as dashed lines in Figure 2 (top-left panel), at $z \sim 15.5$, while it is still an order of magnitude lower in the BHS case. Occasionally, star formation takes place very close to the BH, e.g., $\sim 1 \mathrm{kpc}$ away at $z \sim 14$. The radiative feedback from this event acts to compound the heating effect from the $\mathrm{BH}$ accretion, thus rendering the removal of gas out of the shallow potential well more effective.

The combined stellar and $\mathrm{BH}$ radiative feedback results in an accretion rate that is on average 4 orders of magnitude below the Eddington value at $z=14-13$. Even $300 \mathrm{Myr}$ after $\mathrm{BH}$ formation, the mass of the $\mathrm{BH}$ has increased by only $1.5 \%$ in the BHS simulation, whereas it has grown by two orders of magnitude in the BHN case. This indicates that the feedback from a stellar-mass $\mathrm{BH}$ is sufficiently strong to prevent significant growth, suggesting a very important constraint on $\mathrm{SMBH}$ formation scenarios. We infer that the radiative feedback from an accreting BH might be partly responsible for the low density of quasars at redshifts $z \sim 6$, by suppressing early BH growth.

\section{Conclusions}

We have studied how the assembly of a primordial galaxy is affected by the radiative feedback from an accreting, isolated stellar-mass $\mathrm{BH}$ and an $\mathrm{HMXB}$, which are two possible end products of Pop III star formation. To accomplish this, we have carried out three cosmological simulations which self-consistently account for the radiation from individual Pop III stars, and from a central BH X-ray source. 
We have shown that locally the feedback from an isolated, accreting $\mathrm{BH}$ is very efficient, leading to a strong suppression of the early growth of the seed BH. Without such feedback, the growth rate quickly reaches near-Eddington values. We suggest that the radiative feedback from accreting BHs plays a key role in suppressing early $\mathrm{BH}$ growth, thus constraining models for $\mathrm{SMBH}$ formation.

The feedback from an efficiently radiating HMXB is very strong locally, and moderately important globally. Locally, the effect on the surrounding primordial gas is to heat it to high temperatures of $\gtrsim 10^{4} \mathrm{~K}$, and to fully ionize it. The corresponding strong photo-evaporative outflow suppresses central gas densities, thus preventing any subsequent star formation within the emerging galaxy. Our results imply that once a halo of $\sim 10^{6} M_{\odot}$ harbors an HMXB, the ensuing strong radiative feedback will delay the condensation of gas in the atomic cooling halo, possibly leading to a decrease in the number of first galaxies at a given epoch.

\section{References}

[1] Ricotti, M., Gnedin, N. Y., \& Shull, J. M., The Fate of the First Galaxies. II. Effects of Radiative Feedback, 2002, ApJ, 575, 49

[2] Abel, T., Wise, J. H., \& Bryan, G. L., The H II Region of a Primordial Star, 2007, ApJL, 659, L87

[3] Greif, T. H., Glover, S. C. O., Bromm, V., \& Klessen, R. S., The First Galaxies: Chemical Enrichment, Mixing, and Star Formation, 2010, ApJ, 716, 510

[4] Wise, J. H., Turk, M. J., Norman, M. L., \& Abel, T., The Birth of a Galaxy: Primordial Metal Enrichment and Stellar Populations, 2012, ApJ, 745, 50

[5] Kuhlen, M., \& Madau, P., The first miniquasar, 2005, MNRAS, 363, 1069

[6] Alvarez, M. A., Wise, J. H., \& Abel, T., Accretion onto the First Stellar-Mass Black Holes, 2009, ApJL, 701, 133

[7] Haiman, Z., Thoul, A. A., \& Loeb, A., Cosmological Formation of Low-Mass Objects, 1996, ApJ, 464,523

[8] Yoshida, N., Abel, T., Hernquist, L., \& Sugiyama, N., Simulations of Early Structure Formation: Primordial Gas Clouds, 2003, ApJ, 592, 645

[9] Ricotti, M., \& Ostriker, J. P., X-ray pre-ionization powered by accretion on the first black holes - I. A model for the WMAP polarization measurement, 2004, MNRAS, 352, 547

[10] Madau, P., Rees, M. J., Volonteri, M., Haardt, F., \& Oh, S. P., Early Reionization by Miniquasars, 2004, ApJ, 604, 484

[11] Jeon, M., et al., The First Galaxies: Assembly with Black Hole Feedback, ApJ submitted (arXiv:1111.6305)

[12] Fan, X. et al., A Survey of z > 5.7 Quasars in the Sloan Digital Sky Survey. IV. Discovery of Seven Additional Quasars, 2006, AJ, 131, 1203

[13] Springel, V., The cosmological simulation code GADGET-2, 2005, A\&A, 364, 1105

[14] Greif, T. H., Johnson, J. L., Klessen, R. S., \& Bromm, V., The first galaxies: assembly, cooling and the onset of turbulence, 2009, MNRAS, 399, 639

[15] Bondi, H., \& Hoyle, F., On the mechanism of accretion by stars, 1944, MNRAS, 104, 273 